\title{
On the Role of Hand Synergies in the Optimal Choice of Grasping Forces
}

\author{
Marco Gabiccini* ${ }^{* \dagger}$ and Antonio Bicchi ${ }^{\dagger \ddagger}$ \\ * Dept. of Mechanical, Nuclear and Production Eng. \\ University of Pisa, 56122 Pisa, Italy \\ † Interdept. Research Center "E. Piaggio" \\ University of Pisa, 56122 Pisa, Italy \\ $\ddagger$ IIT - Istituto Italiano di Tecnologia, \\ 16163 Genova, Italy
}

\begin{abstract}
Recent work on the analysis of natural and robotic hands has introduced the notion of postural synergies as a principled organization of their complexity, based on the physical characteristics of the hand itself. Such characteristics include the mechanical arrangements of joints and fingers, their couplings, and the low-level control reflexes, that determine the specific way the concept of "hand" is embodied in a human being or a robot. While the focus of work done so far with postural synergies has been on motion planning for grasp acquisition, in this paper we set out to investigate the role that different embodiments have on the choice of grasping forces, and on the ultimate quality of the grasp. Numerical results are presented showing quantitatively the role played by different synergies (from the most fundamental to those of higher-order) in making a number of different grasps possible. The effect of number and types of engaged synergies on the distribution of optimal grasp forces is considered. Moreover, robustness of results is investigated with respect to variation in uncertain parameters such as contact and joint stiffness.
\end{abstract}

\section{INTRODUCTION}

Recent advances in neuroscience research have shown that the description of how the human hand moves during grasping is dominated by trajectories in a configuration space of much smaller dimension than the kinematic count would suggest. Such configuration space is sometimes referred to as the space of postural synergies, or the eigengrasp space.

One of the explanations for human efficiency in selecting appropriate grasps is that humans somehow unconsciously simplify the large search space through learning and experience. In a developmental perspective, it can be conjectured that learning is applied to a series of inner representations of the hand of increasing complexity, which varies with the experience and the degree of accuracy required. Santello et al. [1] investigated this hypothesis by collecting a large set of data containing grasping poses from subjects that were asked to shape their hands in order to mime grasps for a large set $(N=57)$ of familiar objects. Principal Components Analysis (PCA) of this data revealed that the first two principal components account for more than $80 \%$ of the variance, suggesting that a very good characterization of the recorded data can be obtained using a much lower-dimensional subspace of the hand DoF space. These and similar results seem to suggest that, out of the ca. 20 DoFs of a human hand, only two or three combinations can be used to shape the hand for basic grasps used in everyday life. It might also be speculated that higher order synergies can be recruited for executing more complex tasks, such as adaptive grasp force control, fine manipulation or haptic exploration.

One first explanation of the observed inter-digit coordination could be advanced in terms of mechanical constraints in the anatomy of the hand. More refined approaches recognize the role of peripheral and central nervous systems in establishing sensory-motor control synergies, as discussed, e.g., in [2] and [3]. Currently, investigations in the role and origin of synergies are being actively pursued by neuroscientists.

What the current knowledge about the neurophysiology of human hands already suggests at this point is that the brain uses the hand - meant as a cognitive entity for the organ of the sense of active touch - not as a mere collection of articular joints and muscles, but rather as an organized and ordered ensemble. The organization is dictated by principles that are embedded in the hand's embodiment - i.e. in its physical characteristics such as the mechanical arrangements of joints and fingers, their couplings, and the low-level control reflexes.

These ideas can be brought to use in robotics, as they suggest a new and principled way of simplifying the design and analysis of hands (as opposed to many empirical, often arbitrary design attempts), which has been the main roadblock for research in artificial hands in the past [4].

The application of synergy concepts has been pioneered in robotics by [5] and [6]. In [5], and later on in [7], the idea has been exploited in the dimensionality reduction of the search space in problems of automated grasp synthesis, and has been applied effectively to derive pre-grasp shapes for a number of complex robotic hands. In [6], authors designed a mechanical hand in which more or less accurate actuators are connected to different groups of mechanically interconnected joints, with a priority inspired by resemblance to postural synergies observed in human hands.

Much remains to be done to understand and exploit the implications of the synergy approach to analysis and design of artificial hands. For instance, the role of synergies in fine manipulation and haptic exploration are completely unexplored at present. Very little is known even about all grasping phases subsequent to grasp pre-shaping and contact acquisition, most notably on grasp force distribution and the fundamental problems of form and force closure.

In this paper, we study the effect of the number and type of engaged synergies on the distribution of optimal grasp forces and on the ultimate quality of the grasp.

To investigate grasp force distribution problems in basic whole-hand grasps executed by a hand with a limited number of (synergistic) DoFs, the analytical approach followed by the majority of grasp force studies, which abstract their analysis from the specific physical characteristics of the grasping hand, is unsuitable. We therefore introduce a novel analytical 

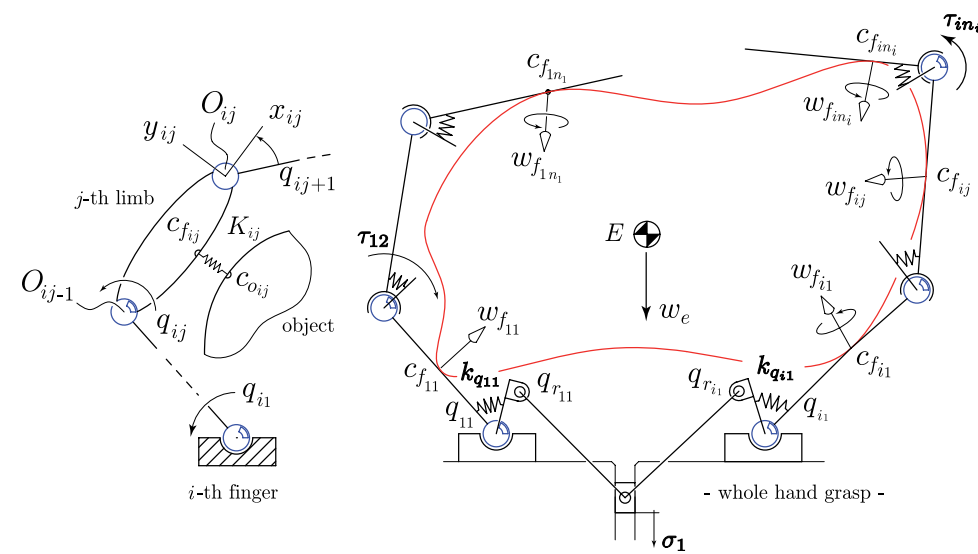

Fig. 1: Schematic of whole-limb manipulation with synergies.

framework, which draws upon previous work on underactuated grasps. Numerical results are presented showing quantitatively the role played by different synergies (from the most fundamental to those of higher-order) in making possible a number of different grasps. As the analysis method we propose to solve force indeterminacies in the rigid-body system introduces a model of compliance in the system, an issue may arise of how strongly our results depend on a fundamentally uncertain and varying parameter such as compliance. To partially address this problem, we show that our numerical results are quite robust with respect to such uncertainties.

\section{Preliminaries: Quasi-STATIC Manipulation MODEL}

\section{A. Rigid Multibody Model}

We model a cooperating manipulation system as a collection of an arbitrary number of robot "fingers" (i.e., simple chains of links connected through revolute or prismatic joints) attached to a common base "palm", and an object, which is in contact with all or some of the links. With reference to Fig. 1 and adopting the notation in Table I, let $k$ be the number of fingers, $n_{i}$ the number of degrees of freedom for the $i^{t h}$ finger, and set $n=\sum_{i=1}^{k} n_{i}$ as the total number of hand degrees of freedom (DoFs). Moreover, let $q \in \mathbb{R}^{n}$ be the vector of joint angles for the whole hand, $q_{i} \in \mathbb{R}^{n_{i}}$ that for the $i^{t h}$ finger, and $q_{i j} \in \mathbb{R}$ the angle of the $j^{\text {th }}$ limb on the $i^{\text {th }}$ finger.

According to standard conventions, we consider a fixed (palm) frame $\mathrm{P}=\left(O_{p} ; x_{p}, y_{p}, z_{p}\right)$ and, for each finger in the hand, we attach a D.-H. frame $\mathrm{S}_{i j}=\left(O_{i j} ; x_{i j}, y_{i j}, z_{i j}\right)$ to its $j^{\text {th }}$ link, a local frame $\mathrm{L}_{i j}=\left(O_{l_{i j}} ; x_{l_{i j}}, y_{l_{i j}}, z_{l_{i j}}\right)$ to the center of the limb and a normalized Gauss frame $\mathrm{C}_{i j}=\left(C_{i j} ; x_{c_{i j}}, y_{c_{i j}}, z_{c_{i j}}\right)$ local to the surface of the fingertip, with its $z$-axis aligned with the outward normal. The frame attached to the object is $\mathrm{E}=\left(E ; x_{e}, y_{e}, z_{e}\right)$, with origin coincident with the center of gravity $G$, i.e., $E \equiv G$.

Since only some of the fingers and/or some of the limbs in each finger may be in contact with the object at point $c_{f_{i j}} \in \mathbb{R}^{3}$, we define $k$ sets of indices $\nu(i),(i=1, \ldots, k)$, each one describing the connectivity of the $i^{t h}$ finger with the object. In view of further analysis, we distinguish between corresponding points $c_{f_{i j}}$ and $c_{o_{i j}}$ on the finger and on the object, respectively. On the contrary, under the hypothesis of small relative elastic rotations, we confuse local frames at the contact point on the object and the limbs with the unique $\mathrm{C}_{i j}$.

\begin{tabular}{r|l}
\hline \multicolumn{1}{|c|}{ Notation } & Definition \\
\hline \hline $\mathrm{P}$ & palm (inertial) frame \\
$\mathrm{S}_{i j}$ & D.-H. limb frame, $i^{\text {th }}$ finger, $j^{t h}$ limb \\
$\mathrm{L}_{i j}$ & local limb frame, $i^{t h}$ finger, $j^{t h}$ limb \\
$\mathrm{C}_{i j}$ & $\begin{array}{l}\text { local contact frame, } i^{t h} \text { finger, } j^{t h} \text { limb } \\
\text { object frame }\end{array}$ \\
\hline$n$ & number of hand joints \\
$q \in \mathbb{R}^{n}$ & actual joint angles \\
$q_{r} \in \mathbb{R}^{n}$ & reference joint angles \\
$\tau \in \mathbb{R}^{n}$ & joint torques \\
$\sigma \in \mathbb{R}^{s}$ & number of postural syergies \\
$\eta \in \mathbb{R}^{s}$ & synergistic displacements \\
${ }^{c}$ & synergistic generalized forces \\
$\xi_{f} \in \mathbb{R}^{c}$ & contact force/torque vector \\
$\xi_{o} \in \mathbb{R}^{c}$ & twists of the contact points on the fingers \\
\hline$u \in \mathbb{R}^{6}$ & twists of the contact points on the object \\
$\xi_{e} \in \mathbb{R}^{6}$ & position and orientation of the object \\
$w_{e} \in \mathbb{R}^{6}$ & object twist \\
\hline$J \in \mathbb{R}^{c \times n}$ & object wrench \\
$S \in \mathbb{R}^{n \times s}$ & hand Jacobian matrix \\
$G \in \mathbb{R}^{6 \times c}$ & synergy matrix \\
grasp matrix
\end{tabular}

TABLE I: Notation for grasp analysis with postural synergies.

In this analysis, the location of the contact points in space is assumed to be known, by either planning or sensing.

Let $f_{i j} \in \mathbb{R}^{c_{i j}}$ be the components in the contact frame $C_{i j}$ of force/torque that can be transmitted through the contact at point $c_{f_{i j}}$. Its dimension depends on the contact type, e.g., $c_{i j}=3$ for Point Contact With Friction (PCWF), $c_{i j}=4$ for Soft Finger (SF), and its contribution to the actual wrench is characterized by the wrench basis $H_{i j}^{T}$ [8].

Adopting the notation introduced in Table I, the balance and congruence equations for the object can be respectively written as

$$
\begin{aligned}
{ }^{p} w_{e} & =G f, \\
\xi_{o} & =G^{T p} \xi_{e},
\end{aligned}
$$

where $G \in \mathbb{R}^{6 \times c}$ is the grasp matrix.

Similarly, the balance and congruence equations for the hand are, respectively,

$$
\begin{aligned}
\tau & =J^{T} f, \\
\xi_{f} & =J \dot{q},
\end{aligned}
$$

where $J \in \mathbb{R}^{c \times n}$ is the hand Jacobian.

For the reader's convenience, a detailed derivation of eqs. (1)-(4) can be found in the Appendix.

\section{B. Introducing Elasticity}

Now, with reference to taxonomy in [9], in order to handle statically-indeterminate or hyperstatic grasps, which occur when $\mathcal{N}(G) \cap \mathcal{N}\left(J^{T}\right) \neq 0$, we follow [10], and introduce a set of virtual springs at the interface between corresponding contact points $c_{f_{i j}}$ and $c_{o_{i j}}$ on the fingers and the object. These result in a system of linear constitutive equations linking the components of relative displacements $\delta \xi_{o f}:=\delta \xi_{o}-\delta \xi_{f}$, that violate the constraints, to the corresponding contact force

$$
f=f_{0}+\delta f, \quad \delta f=K \delta \xi_{\text {of }},
$$

where $f_{0}$ is the contact force in the reference configuration $\delta \xi_{o}=\delta \xi_{f}=0$. According to [11], the stiffness matrix $K \in$ $\mathbb{R}^{c \times c}$ can be computed as

$$
K=\left(C_{s}+J C_{q} J^{T}\right)^{-1},
$$




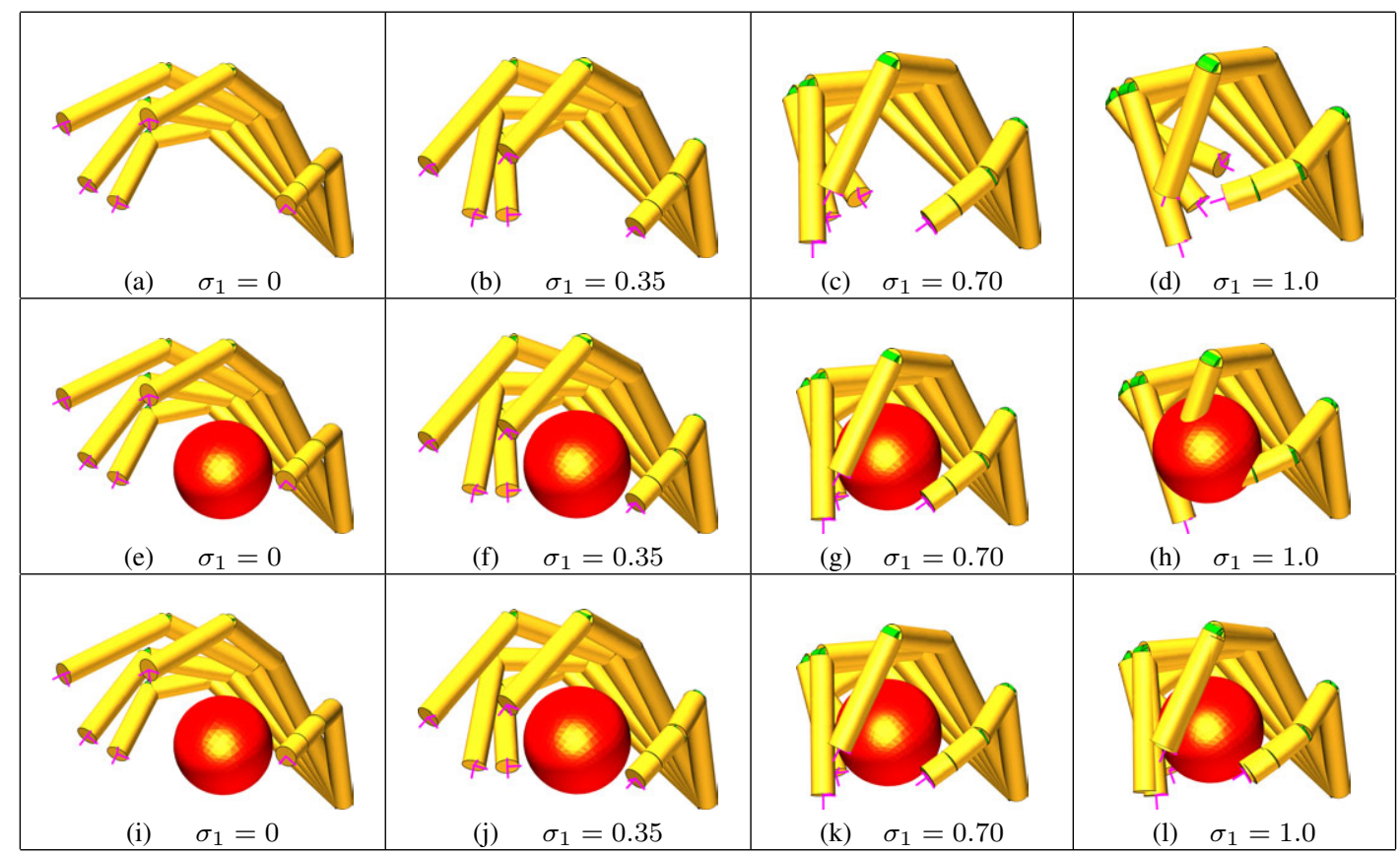

Fig. 2: Effect of the first synergy $S_{1}$ on the hand posture as a function of the normalized synergy coefficient $\sigma_{1} \in[0,1]$. Figs. (a)-(d): reference motion of the hand. Figs. (e)-(h): motion of the hand without contact interaction. Figs. (i)-(l): motion of the hand with contact interaction and joint elasticity.

where $C_{s} \in \mathbb{R}^{c \times c}$ is the structural compliance matrix (due to, e.g., the flexibility of limbs and fingerpads), and with $C_{q} \in \mathbb{R}^{n}$ the diagonal matrix whose element in position $(k, k)$ is the compliance at the $k$-th joint. Joint compliance in animals is determined by the elastic properties of muscles and tendons and by modulation of the stretch reflex. Similar roles in robot hands are played by transmission and actuator compliance, and by the gain of the $k^{t h}$ position servo. It should be noticed that in both cases joint compliance can be varied, both intentionally and not, although not necessarily in an independent way from joint to joint. The matrices $C_{s}$ and $C_{q}$ employed for the numerical tests reported in this paper are of the form

$$
C_{s}=\left(1 / k_{\mathrm{stru}}\right) I_{c \times c}, \quad C_{q}=\left(1 / k_{\mathrm{ss}}\right) I_{n \times n},
$$

where $k_{\text {stru }}(\mathrm{N} / \mathrm{mm})$ is a value of structural stiffness, while $k_{\mathrm{ss}}(\mathrm{Nmm} / \mathrm{rad})$ is an admissible value of joint stiffness.

\section{Formulation OF THE GRASPING PROBLEM FOR THE HAND EMBODIED}

\section{A. The "Softly Underactuated" Model}

Consideration of synergies introduces a new vista on the grasp problem. A direct interpretation of the results described in [1] would imply that the joint configuration vector $q$ could be represented as a function of fewer elements, collected in a synergy vector $\sigma \in \mathbb{R}^{s}$, as $q=q(\sigma)$, which effectively constraints the hand configuration in an $s$-dimensional submanifold of the joint configuration manifold. Synergistic hand velocities would belong to the tangent bundle to this manifold, and could be locally described by a linear map $\dot{q}=S(\sigma) \dot{\sigma}$. This situation is illustrated in Fig. 2, panels (a)-(d), where the reference posture of the hand is reported as a function of the synergy coefficient $\sigma_{1}$ scaling the first synergy vector $S_{1}$.

However, the sole kinematic model of the hand fails to describe the actual grasp of an object (Fig. 2, panels (e)- (h)). Therefore, contact forces must be brought into play if a realistic grasp analysis is in order.

Taking a step further, and in view of dealing with the most general case of statically-indeterminate grasps, both contact and joint compliances have to be included in the analysis.

Therefore, in the model we propose, the synergistic hand displacements $\delta \sigma \in \mathbb{R}^{s}$ do not command the joint displacements $\delta q \in \mathbb{R}^{n}$ directly, as assumed in the analysis in [7] and implemented in the design in [6]. Instead, the synergistic displacements input $\delta \sigma$ command the joint reference positions $q_{r}$, as described by the following linear equations (see also Fig. 1)

$$
\delta q_{r}=S \delta \sigma, \quad S \in \mathbb{R}^{n \times s}(1 \leq s \leq n),
$$

which, in turn, are related to the actual joint displacements by the constitutive equation

$$
\delta q=\delta q_{r}-C_{q} \delta \tau
$$

where $C_{q}$ is the joint compliance introduced in (7). It is worth mentioning that this model, whereby motion is controlled by a reference position and modulation of joint stiffness, has apparent similarities with the equilibrium point hypothesis in the motor control literature [12].

In the pre-grasp phase, forces are null $\delta \tau=0$, hence $\delta q=$ $\delta q_{r}$ and the reference and actual posture overlap perfectly (first three columns in Fig. 2). Hence, in this approach phase, the rigid synergy model $q=q(\sigma)$ is valid. When an actual grasp of an object occurs, however, the interference (contact) forces and hand compliance cause the actual hand to deviate from the reference hand (panel (1) in Fig. 2). Thus, in our hand model, the actual hand configuration is driven by synergies, but modifies its posture according to the object shape and compliance. We denote this as a soft synergy model of hands.

In the following of this paper, we employ data recorded in [1] and the definition of finger coordination patterns defined 
through PCA, to obtain numerical values for the synergy matrix $S$ (aka "eigengrasp matrix", [7]). More specifically, different simplified synergy matrices $S$ can be obtained extracting a number of columns from the orthogonal full synergy matrix $S \in \mathbb{R}^{n \times n}$ obtaned from PCA data, and whose columns are ordered in an increasing order with respect to the relative contribution to the variance. Each of these extracted synergy matrices will represent a model of a specific (underactuated) hand.

For later use, let us further introduce the balance equation

$$
\eta:=S^{T} \tau=S^{T} J^{T} f,
$$

where $\eta \in \mathbb{R}^{s}$ are the generalized synergistic forces corresponding to synergistic displacements.

We now shift our attention onto the effect of introducing motion coordination patterns for fingers on the ability of the hand to exert internal forces actively (full derivation of the analytical results is omitted for brevity).

B. General Solution of the Grasping Problem With Synergies

Extending the work [10], it can be shown that for a general grasping system with elastic contacts that applies a wrench $w_{e}$ to the object, the general solution to the force distribution problem is given by

$$
f=G_{K}^{R} w_{e}+\delta f_{h r_{s}}+\delta f_{h o_{s}}, \quad \delta f_{h r_{s}}=E_{s} y, \delta f_{h o_{s}}=P_{s} z,
$$

where $G_{K}^{R}=K G^{T}\left(G K G^{T}\right)^{-1} \in \mathbb{R}^{c \times 6}$ is the $K$-weighted pseudoinverse of $G$, providing the particular solution $G_{K}^{R} w_{e}$ that minimizes the potential energy $\frac{1}{2} \delta \xi_{o f}^{T} K \delta \xi_{\text {of }}$, (see [13], [14]). In eq. (11), the columns of matrix $E_{s} \in \mathbb{R}^{c \times e_{s}}$ form a basis for the range space of the matrix $F_{s} \in \mathbb{R}^{c \times s}$, mapping the $\delta \sigma$ 's into the active internal forces $\delta f_{h r_{s}}$ that can be commanded by synergistic displacements

$$
\delta f_{h r_{s}}=F_{s} \delta \sigma, \quad F_{s}:=F S, \quad \operatorname{rank}\left(F_{s}\right)=e_{s} .
$$

The matrix $F \in \mathbb{R}^{c \times n}$ has expression

$$
F:=\left(I-G_{K}^{R} G\right) K J, \quad \operatorname{rank}(F)=e,
$$

and maps independently controlled joint reference displacements $\delta q_{r}$ 's into active internal forces

$$
\delta f_{h r}=F \delta q_{r}
$$

It is worth noting that if all the DoFs of the hand are independently controlled, $\delta q_{r}=\delta \sigma, S=I \in \mathbb{R}^{n \times n}$, and eq. (12) reduces to eq. (14).

The expression for $\delta f_{h r_{s}}$ in eq. (11) is a parameterized version of the active homogeneous solution: optimal grasp force distributions can be found by minimizing a cost function with respect to $y \in \mathbb{R}^{e_{s}}$.

The third term $\delta f_{h o_{s}}$ in eq. (11) is a fixed homogeneous solution representing internal, passive (preload) contact forces: this corresponds to contact forces that are preloaded at the beginning of the grasp operation (in the rest of the paper it will be assumed $\left.z=0 \in \mathbb{R}^{p_{s}}\right)$. Matrix $P_{s} \in \mathbb{R}^{c \times p_{s}}$ represents a basis for this subspace. When all the hand joints are independently controlled, the basis matrix is denoted by $P \in \mathbb{R}^{c \times p}$, where $p$ is the dimension of this subspace.

We summarize the above results by introducing the following subspaces

$$
\begin{aligned}
\mathcal{F}_{h} & =\mathcal{R}(A)=\mathcal{N}(G) \subset \mathbb{R}^{c}, \\
\mathcal{F}_{h r_{s}} & =\mathcal{R}\left(E_{s}\right)=\mathcal{N}(G) \cap\left(\mathcal{R}(K J S)+\mathcal{R}\left(K G^{T}\right)\right), \\
\mathcal{F}_{h o_{s}} & =\mathcal{R}\left(P_{s}\right)=\mathcal{N}(G) \cap \mathcal{N}\left((J S)^{T}\right),
\end{aligned}
$$

that yields the natural decomposition

$$
\mathcal{F}_{h_{s}}=\mathcal{F}_{h r_{s}} \oplus \mathcal{F}_{h_{s}}, \quad \mathbb{R}^{c}=\mathcal{F}_{h_{s}} \oplus \mathcal{R}\left(G_{K}^{R}\right)
$$

In case of independent joint control $\left(S=I \in \mathbb{R}^{n \times n}\right)$, active and passive subspaces in eqs. (15) reduce to $\mathcal{F}_{h r}$ and $\mathcal{F}_{h o}$, respectively, as in [10].

It is worth noting that among the free vectors $y \in \mathbb{R}^{e_{s}}$, $\hat{y}$ will denote a particular choice corresponding to an optimal grasp force distribution with respect to a chosen cost function.

From a computational point of view, the calculation of the desired basis matrix $E_{s}=\operatorname{colbasis}\left(F_{s}\right)$ from eq. (12) is not optimal, since it entails the explicit calculation of $G_{K}^{R}$. A more efficient algorithm can be obtained by intersection of subspaces, observing that a consistent set of internal forces, parameterized by $x \in \mathbb{R}^{h}$, synergy displacements $\delta \sigma \in \mathbb{R}^{s}$, and object motions $\delta \xi_{e} \in \mathbb{R}^{6}$, must belong to the nullspace of $Q_{s} \in \mathbb{R}^{c \times(h+s+6)}$ (whose nullity is $b_{s}$ ), i.e.,

$$
\underbrace{\left[\begin{array}{lll}
A & -K J S & K G^{T}
\end{array}\right]}_{Q_{s}}\left[\begin{array}{c}
x \\
\delta \sigma \\
\delta \xi_{e}
\end{array}\right]=0
$$

Defining $B_{s} \in \mathbb{R}^{(h+s+6) \times b_{s}}$, such that $\mathcal{R}\left(B_{s}\right)=\mathcal{N}\left(Q_{s}\right)$, and partitioning $B_{s}$ as

$$
B_{s}=\left[\begin{array}{lll}
B_{s_{1}}^{T} & B_{s_{2}}^{T} & B_{s_{3}}^{T}
\end{array}\right]^{T},
$$

where $B_{s_{1}} \in \mathbb{R}^{h \times b_{s}}, B_{s_{2}} \in \mathbb{R}^{s \times b_{s}}$, and $B_{s_{3}} \in \mathbb{R}^{6 \times b_{s}}$, such that

$$
\left[\begin{array}{lll}
x^{T} & \delta \sigma^{T} & \delta \xi_{e}^{T}
\end{array}\right]^{T}=\left[\begin{array}{lll}
B_{s_{1}}^{T} & B_{s_{2}}^{T} & B_{s_{3}}
\end{array}\right]^{T} \gamma, \quad \gamma \in \mathbb{R}^{b_{s}},
$$

the subspace of active internal forces can be profitably obtained as

$$
\mathcal{F}_{h r_{s}}=\mathcal{R}\left(A B_{s_{1}}\right), \quad E_{s}:=\operatorname{colbasis}\left(A B_{s_{1}}\right),
$$

The synergy displacements $\delta \hat{\sigma}$ that must be commanded if a desired internal force $\delta \hat{f}=E_{s} \hat{y}$ is to be applied are given by

$$
\delta \hat{\sigma}=B_{s_{2}}\left(A B_{s_{1}}\right)^{+} E_{s} \hat{y}
$$

According to eq. (8) and eq. (23), the joint reference position is displaced by

$$
\delta q_{r}=S B_{s_{2}}\left(A B_{s_{1}}\right)^{+} E_{s} \hat{y}
$$

and the object moves to a new equilibrium position defined by

$$
\delta \xi_{e}=B_{s_{3}}\left(A B_{s_{1}}\right)^{+} E_{s} \hat{y}
$$

Due to contact forces in the interaction with the object and the joint compliance, the hand joints moves differently with respect to the commanded reference, i.e., $\delta q \neq \delta q_{r}$. Their values can be explicitly calculated as

$$
\delta q=\left[I-C_{q} J^{T}\left(I-G_{K}^{R} G\right) K J\right] S \delta \hat{\sigma},
$$

where $\delta \hat{\sigma}$ can be recovered from (23). It is worth observing that in case of perfectly rigid joints, $C_{q}=0 \in \mathbb{R}^{n \times n}$, and $\delta q=\delta q_{r}$. The other quantities are also modified due to the increased global stiffness, as evident from the definition of $K$ in (6).

Finally, corresponding variation of the joint torques $\delta \tau$ can be obtained as

$$
\delta \tau=J^{T}\left(I-G_{K}^{R} G\right) K J S \delta \hat{\sigma},
$$

and the associated variation of the synergistic forces $\delta \eta$ is

$$
\delta \eta=S^{T} \delta \tau
$$




\section{Qualitative Analysis of Hand Embodiment}

With reference to eq. (12), the following relationships between ranks hold

$$
\begin{aligned}
& \underbrace{\operatorname{rank}\left(F_{s}\right)}_{e_{s}}=\underbrace{\operatorname{rank}(S)}_{s}-\operatorname{dim}(\mathcal{N}(F) \cap \mathcal{R}(S)) \\
& \operatorname{rank}\left(F_{s}\right) \leq \min \{\operatorname{rank}(F), \operatorname{rank}(S)\} \\
& \operatorname{rank}\left(F_{s}\right) \geq \operatorname{rank}(F)+\operatorname{rank}(S)-n
\end{aligned}
$$

Therefore, under the condition that each synergy $S_{i}\left(i \in \mathcal{S}^{1}\right)$ has a non-null projection onto $\mathcal{R}\left(F^{T}\right)$, we can assume that $e_{s}=s$. In this case, consider a fully actuated grasp with $\operatorname{rank}(F)=e$. For the same grasp, consider underactuation and increase one by one the number of the engaged columns of $S$. As the number of synergies engaged increases, say $1 \leq s \leq e$, the dimension of the subspace $\mathcal{F}_{h r_{s}}$ of active internal forces also increases in the same manner. For $s>e$, according to eq. (30), $e_{s}=e$, and the dimension of the subspace $\mathcal{F}_{h r_{s}}$ reaches a plateau. This means that for fully actuated grasping systems characterized by an $e$-dimensional $\mathcal{F}_{h r}$, underactuation with a number of synergies $s \sim e$ does not endanger the ability to exert the same internal forces. If $e$ is "small", say e.g. $s=2$ or $s=3$, application of an equally "small" number of synergies $s$ results in a great control simplification without side effects on the grasping ability of the system, see results in sec. V-C.

A fundamental issue we want to investigate is the link between basic synergies, i.e., the first components obtained via PCA, accounting for much of the variance in geometric posture space, and the ability of the corresponding underactuated system to firmly grasp an object. However, the sole rank count in eq. (29) does not allow to compare quantitatively synergies with different shapes, i.e., different columns of $S$. Therefore, in this analysis the behaviour of a system underactuated by means of different number of synergies and, once fixed their number, with different synergies $S_{i}$, is presented. The performance parameter is the ability to attain force-closure conditions and, if this is the case, in obtaining lower values of a suitably defined cost function in determining optimal grasping forces. Then, we discuss robustness issues with respect to variations of the grasping stiffness: the range, going from values typical of robotic hands to those of the human hand, is elicited from [15], and [16].

\section{Force-Closure Problem with Synergies}

At an intuitive level, the meaning of "force-closure" is that motions of the grasped object are completely restrained against arbitrary external disturbances, by virtue of the contact forces that the hand is capable to exert on the object. We emphasize here that this definition is very relevant to synergistically (under)actuated hands. Indeed (as opposite to the purely geometric nature of form-closure), force-closure involves consideration of which contact forces can be actively applied on the object by the specific hand under consideration. Under this regard, it clearly makes a difference if an object is grasped by a hand controlled by different numbers and types of synergies (corresponding to different active internal force subspaces, as discussed in the previous section).

Accordingly, we adopt here the definition of force-closure given in [17], which considers the case of underactuated hands:

${ }^{1} \mathcal{S}$ is a set of indices used to select the corresponding columns in $\bar{S}$ to build $S$.
Definition 1 (Force-Closure): A grasp is defined ForceClosure if and only if the following conditions are satisfied:

1. forces in arbitrary directions are resistible, i.e. $\operatorname{rank}(G)=6$ 2. the hand configuration is prehensile, i.e. $\exists y$ such that $f(y) \in \operatorname{Int}(\mathcal{F})^{2}$, with $f(y)=E y$

In Definition $1, \mathcal{F}$ is the composite friction cone defined as $\mathcal{F}=\mathcal{F}_{11} \times \cdots \times \mathcal{F}_{k, n_{k}}$. For brevity, we recall here only those types of friction cone implemented in our software, used to carry out numerical results in later sections. For a point contact with friction (PCWF), we have $f_{i j} \in \mathbb{R}^{3}$ and

$$
\mathcal{F}_{i j}=\left\{f_{i j} \in \mathbb{R}^{3} \mid f_{i j_{3}} \geq 0, \frac{1}{\mu_{i j}}\left(f_{i j_{1}}^{2}+f_{i j_{2}}^{2}\right) \leq f_{i j_{3}}^{2}\right\},
$$

where $f_{i j_{3}}$ is the normal component of the contact force at the point of contact $c_{i j}, f_{i j_{1}}, f_{i j_{2}}$ the components in the tangential directions, and $\mu_{i j}$ the Coulomb friction coefficient.

For a soft-finger with elliptical friction limit approximation [18], we have $f_{i j} \in \mathbb{R}^{4}$ and

$\mathcal{F}_{i j}=\left\{f_{i j} \in \mathbb{R}^{4} \mid f_{i j_{3}} \geq 0, \frac{1}{\mu_{i j}}\left(f_{i j_{1}}^{2}+f_{i j_{2}}^{2}\right)+\frac{1}{\mu_{i j_{t}}} f_{i j_{4}}^{2} \leq f_{i j_{3}}^{2}\right\}$,

where $\mu_{i j_{t}}$ is a proportionality constant between the torsion and shear limits. It is worth noting that the above models do not necessarily assume equal friction coefficients at all contact points: however, for brevity, in the numerical tests reported in sec. V-C and V-D, the friction coefficients will be assumed constant, i.e., $\mu_{i j}=\mu$ and $\mu_{i j_{t}}=1$.

In view of formulating and solving the problem as a second order cone programming (SOCP) one, for which efficient algorithms and interfaces are today a mature technology, we recall that the satisfaction of the friction limit constraints in eq. (32) and (33) is equivalent to the positive definiteness of matrix $P=\operatorname{Blockdiag}\left(P_{11}, \ldots, P_{k, n_{k}}\right), P \succ 0$, where the explicit expression for each $P_{i j}$, with $(i=1, \ldots, k ; j \in \nu(i))$, can be found in [18].

Then, consider the problem of finding the optimal distribution of contact forces $f$ in the grasp of an object subject to the external load with regard to the minimization of a suitable cost function $\Psi(y)$. To formalize this problem, we give the following definition:

Definition 2 (Grasping Force Optimization): Given a grasp characterized by $G_{W}^{R}, E_{s}$, and $P_{s}$, and an object wrench $w_{e} \in$ $\mathbb{R}^{6}$, find $\hat{y}$ in eq. (11), such that $f(\hat{y}) \in \operatorname{Int}(\mathcal{F})$, and the cost function $\Psi(f(\hat{y}))$ is minimized.

In numerical tests, we assume: (i) zero preload at the beginning of the grasp ( $z=0$ in eq. (11)); (ii) zero net wrench applied to the object, $w_{e}=0$; (iii) an auxiliary constraint on the minimum value for all the normal components $f_{n}$ of the contact force. Under these hypotheses, the grasping force optimization problem is set up in the following way

$$
\begin{aligned}
& \hat{y}=\operatorname{argmin} \Psi(y) \\
& \text { subject to } f=E_{s} y, \quad P(f) \succ 0, \quad f_{n} \succeq f_{\min }
\end{aligned}
$$

Optimal contact force distribution is sought by employing, in turn, each of the following cost functions

$$
\Psi_{f}(y):=\|f(y)\|_{2}, \quad \Psi_{\tau}(y):=\|\tau(y)\|_{2}, \quad \Psi_{\eta}(y):=\|\eta(y)\|_{2},
$$

\footnotetext{
${ }^{2} \operatorname{Int}(\mathcal{F})$ denote the internal part of the composite friction cone $\mathcal{F}$.
} 
where $f \in \mathbb{R}^{c}$ are the contact forces, $\tau \in \mathbb{R}^{n_{c}}$ the joint torques for the contacting fingers in eq. (3), and $\eta \in \mathbb{R}^{s}$ the synergistic forces in eq. (10).

The problem is set up and solved as a semidefinite program (SDP) by employing the CVX modeling system for convex optimization based on MATLAB, see [19] and [20] for further details. The solver used is SDPT3 [21], which implements an infeasible path-following algorithm for solving general SQLP - conic optimization problems involving semidefinite, secondorder and linear cone constraints.

\section{NUMERICAL TESTS}

\section{A. Paradigmatic Hand Model}

The paradigmatic hand model is schematically represented in Fig. 3. This is the very same model for which detailed data were collected and presented in [1]. The fact that the large amount of high-quality data taken in [1] was kindly made available to us is a reason for this choice, along with the motivation that synergies can be defined for no other kinematic structure without a dose of arbitrarity.

The model has 15 DoFs corresponding to: 4 DoFs for the thumb: TR, TA, TM, TI (Thumb Rotation, Abduction, Metacarpal, Interphalangeal); 3 DoFs for the index: IA, IM, IP (Index Abduction, Metacarpal, Proximal interphalangeal); 2 DoFs for the middle: MM, MP (Middle Metacarpal, Proximal interphalangeal); 3 DoFs for the ring: RA, RM, RP (Ring Abduction, Metacarpal, Proximal interphalangeal); 3 DoFs for the little: LA, LM, LP (Little Abduction, Metacarpal, Proximal interphalangeal). It is worth noting that the middle finger has no abduction since it is considered the "reference finger" in the sagittal plane of the hand. Moreover, the Distal Interphalangeal (DI) angle is not present in none of the four fingers due to the limitation in the sensors embedded in the measuring glove employed, see [1] and references therein. With $\mathrm{P}=\left(O_{p} ; x_{p}, y_{p}, z_{p}\right)$ with indicate the palm frame, and with $S_{i j}=\left(O_{i j} ; x_{i j}, y_{i j}, z_{i j}\right)$ and $\mathrm{C}_{i j}=\left(C_{i j} ; x_{c_{i j}}, y_{c_{i j}}, z_{c_{i j}}\right)$ the D.-H. and the normalized Gauss frame, respectively, for

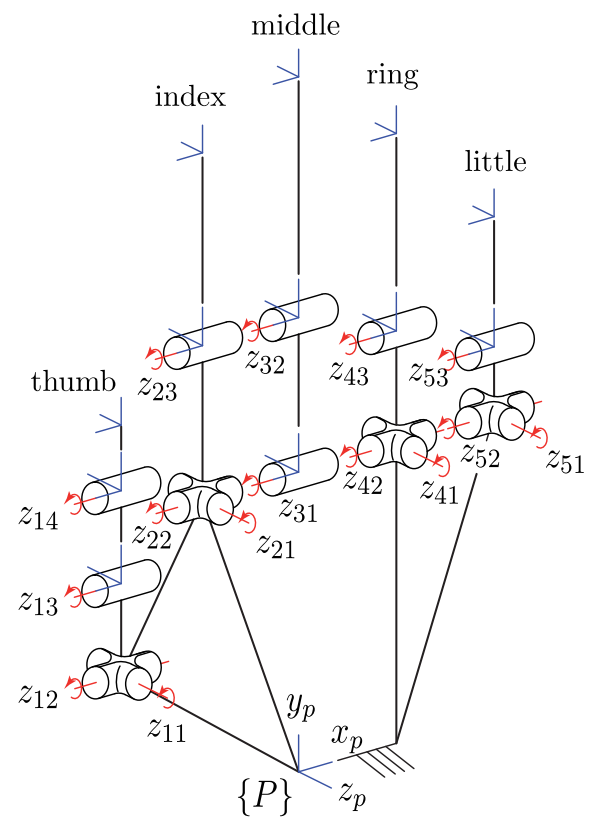

Fig. 3: 15 DoFs kinematic model of the paradigmatic hand.

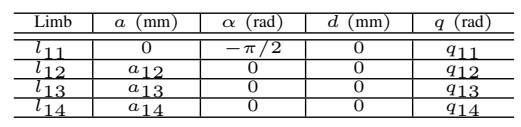

(a) D.-H. table for the Thumb finger.

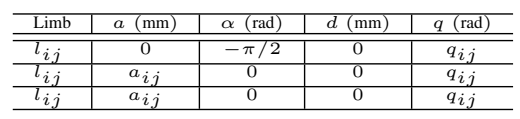

(b) D.-H. table for Index, Ring and Little fingers. Indices take the following values: $i=$ $2,4,5$ and $j=1,2,3$.

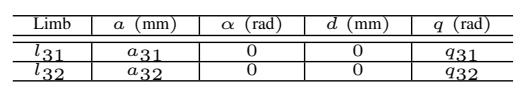

(c) D.-H. table for the Middle finger.

TABLE II: D.-H. tables for the 15 DoFs paradigmatic hand.

the $j^{\text {th }}$ limb on the $i^{\text {th }}$ finger. The D.-H. tables for each finger/group of fingers are shown in Table IIa-IIc.

\section{B. Assumptions in the Definition of Grasp Layouts}

To define possible grasp configurations, we rely on data reported in [1]. In that work, no measurement of the object position, nor spatial location of the contact points, was registered (since the subjects were asked to shape their hands in order to mime grasps, with no physical object present). Therefore we made the following assumptions. Firstly, the hand configuration relative to the imagined grasp of an object, say a cherry, was defined as the mean joint configuration vector recorded in the grasp of that object, among a total of five trials. Therefore, the configuration of the hand is identified with the name of the object grasped. Secondly, a reasonable position of the center of gravity $G$ for the grasped object was defined, taking into account both the hand configuration previously defined and a reasonable shape for that object. Then, the candidate contact point $c_{f_{i j}}$ on each limb $l_{i j}$ was found as the nearest point on the same limb to $G$. Last step was to define the final grasp by selecting the limbs in contact, according to both the shape of the hand and the position of the object relative to the hand the same grasp suggests. The numerical data common to all tests are listed in Tab. III.

\section{Precision grasp - The Cherry}

As a first test case we consider the grasp of a cherry. The hand configuration, the estimated position of the center of gravity $G$ of the object and the contact points are represented

\begin{tabular}{c|c|c|c}
\hline Net Wrench $w_{e}$ & Contact type & Frict. coeff. $\mu$ & $f_{\min }(N)$ \\
\hline \hline 0 & PCWF & 1.5 & 0.1 \\
\hline
\end{tabular}

TABLE III: Nominal data common to all tests.

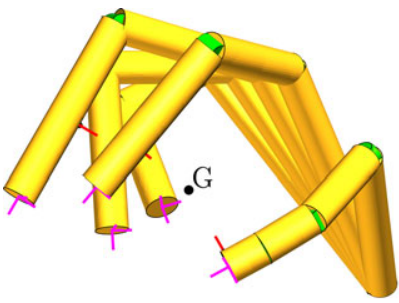

(a) Grasp of the Cherry

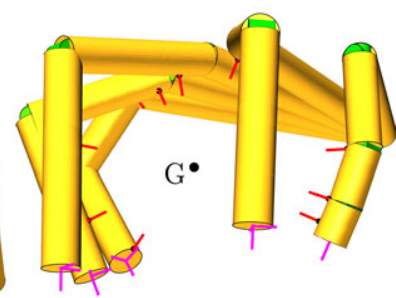

(b) Grasp of the Ashtray
Fig. 4: Hand postures analyzed. 
in Fig. 4 (a). The contact parameters employed are listed in Table III and, with reference to eqs. (6) and (7), the numerical value of the stiffness matrix $K$ is specified by setting $k_{s t r u}=1$ $\mathrm{N} / \mathrm{mm}$ and $k_{\mathrm{ss}}=100 \mathrm{Nmm} / \mathrm{rad}$. For this case, $\operatorname{rank}(G)=6$, $c=9$ and, with reference to eqs. (12), (13), and (15), $h=e=3, p=0$. The optimal contact force distribution is found with respect to $y$ minimizing $\psi_{f}(y)=\|f(y)\|_{2}$.

Let us concentrate on the black curve in Fig. 5a. Interestingly enough, this grasp is force-closure even when engaging only the first synergy $\left(S_{1}\right.$, first column of $S$ ), for which an optimal value of $\|f\|_{2}=0.349 \mathrm{~N}$, is found. When more synergies are engaged in the grasp, the norm of the contact force decreases as the dimension $e_{s}=s$ of $\mathcal{F}_{h r_{s}}$ increases, and there is a higher dimensional space where the optimal solution can be sought. Then, a plateau is obtained once the dimension $e_{s}$ reaches $e$, as confirmed by rank considerations in eq. (29). Therefore, as far as controllable internal contact forces are concerned, no improvement can be obtained in the quality of the grasp by engaging a number of synergies $s>e$.

It is worth stressing that the trend in Fig. 5a is highly dependent on which synergies are considered. Had we engaged synergies in a backward fashion, i.e., from $S_{15}$ to $S_{1}$, we would have obtained a completely different trend. Put another way, if we plug in only one synergy at a time, the grasp is forceclosure only for synergies $S_{1}, S_{3}$ and $S_{4}$, meaning that maybe the first synergies are more fundamental to grasp objects than those of higher-order, but not in a strictly ordered fashion. However, we have no ambition of drawing general conclusions here, since fundamental roles are played by location of the contact points, surface normals, and types of contact constraints, which are here only reasonably estimated.

In order to test the robustness of the above trends, the previous analyses are repeated for different values of $K$ obtained varying $k_{\mathrm{ss}}$ and $k_{\text {stru }}$, as shown in Figs. 5a-5b.

Interestingly, if we select $\Psi_{\eta}(y)=\|\eta\|_{2}$ as cost function, we obtain the trends depicted in Fig. 6a-6b. These show that, in terms of synergistic forces, adding more synergies than those strictly necessary to fulfill the dimension of $\mathcal{F}_{h r}$ worsens the cost, as the range of $S$ (where we project the same $\tau$ 's, see eq. (10)) increases. In other words, if a synergy is not actuated, synergistic forces in its direction are absorbed by the mechanical structure directly, and are not reflected in actuation costs.

Minimization of $\Psi_{\tau}(y)=\|\tau\|_{2}$ bears trends similar to those of Fig. 5, and are here omitted for brevity.

\section{Power Grasp - The Ashtray}

As second test case we consider the grasp of an ashtray. The hand configuration, the estimated position of the center of gravity $G$ of the object and the contact points are represented in Fig. 4 (b). The contact parameters and the nominal stiffness values are the same employed in the previous case. This time, $\operatorname{rank}(G)=6, c=33$ and, with reference to eqs. (12), (13), and (15), $h=27, e=15, p=12$. As intuitive, since $h>$ $e=n$, increasing the number of synergies engaged results in a monotone decrease of the cost function, as elicited from Fig. 7, no matter the values of the grasping stiffness $K$. All curves present a rapid decrease for $1 \leq s \leq 3$, then a lighter decrease for $3<s \leq 6$, after which no practical improvement is registered. Also interesting is that synergy $S_{1}$ represents the only "direction" along which we can obtain force-closure conditions by employing only a 1-dimensional subspace of $\mathbb{R}^{15}$, at least for nominal values of the parameters. No other synergy shows this property. To give an idea, to obtain forceclosure without $S_{1}$, we need a basis composed of columns $S_{2}$

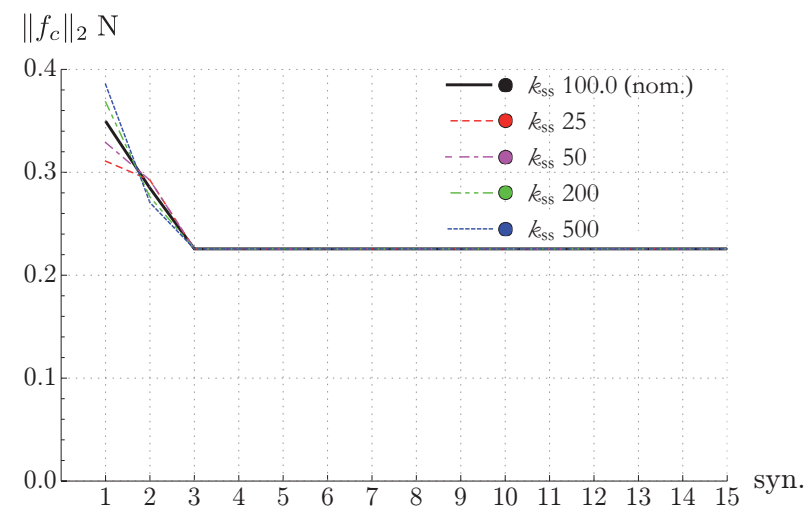

(a) Trends for variation of the steady-state gain $k_{\mathrm{ss}}(\mathrm{Nmm} / \mathrm{rad})$.

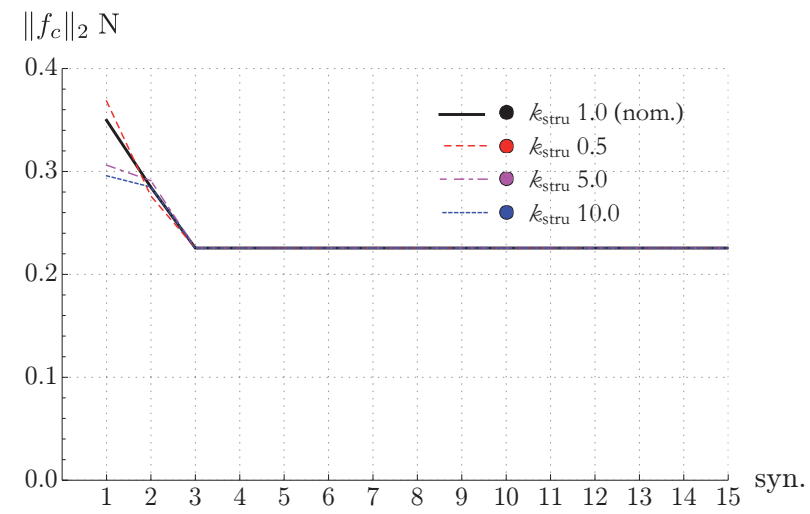

(b) Trends for variation of the structural stiffness $k_{\text {stru }}(\mathrm{N} / \mathrm{mm})$.

Fig. 5: The Cherry. Norm of the optimal contact force $f$ w.r.t. the cost function $\|f\|_{2}$, with increasing number of synergies.

through $S_{9}$, that is a subspace seven-dimensional higher than before.

From Fig. 7, it is interesting to note that a wide variation of $K$ can render infeasible the grasp with only $S_{1}$ engaged. However, adding just another column, e.g. $S_{2}$, solves already the problem.

Finally, if we select $\Psi_{\eta}(y)=\|\eta\|_{2}$ as cost function, we obtain the trends depicted in Fig. 8a-8b.

\section{CONCLUSION}

The force decomposition and optimization problems in multiple whole-limb manipulation of hands with embodied synergies require an extension of existing analytical methods, and the consideration of compliance in the hand-object system.

We have presented two numerical case studies to characterize the role of different postural synergies in the ability of the hand to obtain force-closure grasps. The two case studies addressed a precision grasp and a power grasp, respectively, and are to be considered representative of a number of similar experiments, which could not be reported for space limitations.

The main results obtained from our investigations can be summarized as follows.

The force-closure property of grasps strongly depend on which synergies are used to control the hand. The first few synergies (the first one for the two case studies reported) are sufficient to establish force-closure. If the first few synergies are not actively controlled, force closure can only be obtained if many more DoFs (corresponding to higher-order synergies) are actuated. 


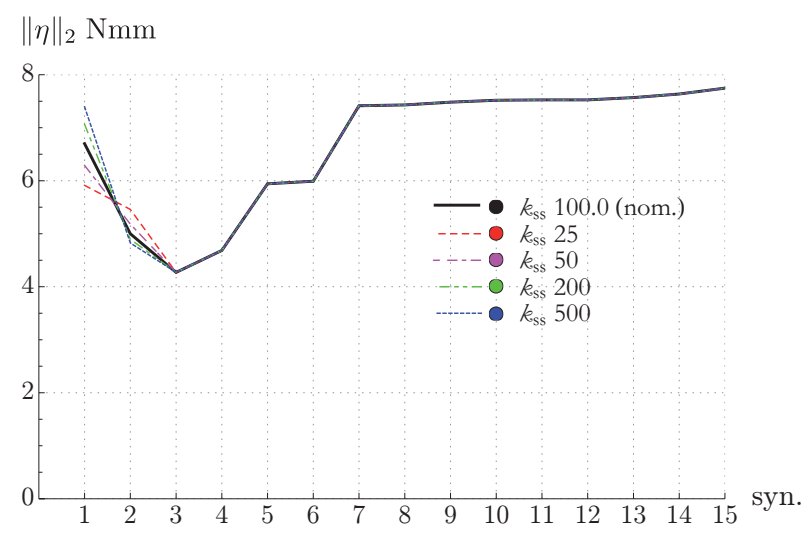

(a) Trends for variation of the steady-state gain $k_{\mathrm{ss}}(\mathrm{Nmm} / \mathrm{rad})$.

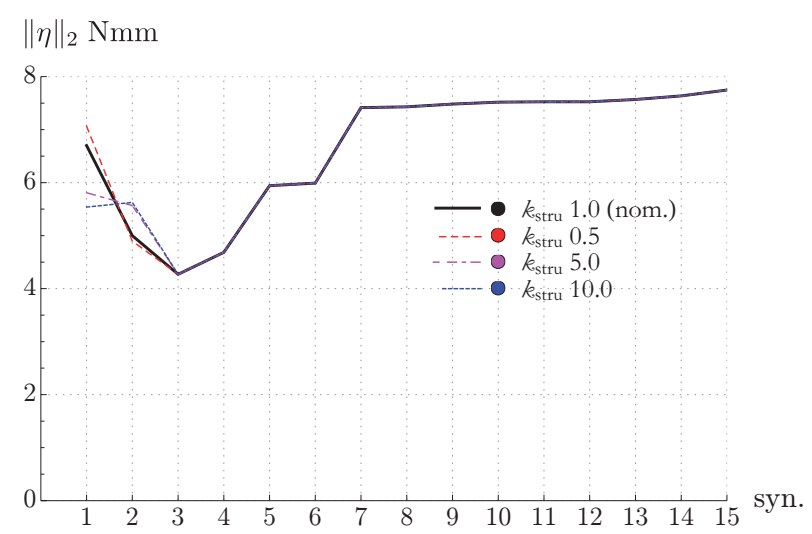

(b) Trends for variation of the structural stiffness $k_{\text {stru }}(\mathrm{N} / \mathrm{mm})$.

Fig. 6: The Cherry. Norm of the optimal synergistic force $\eta=$ $S^{T} \tau$ w.r.t. the cost function $\|\eta\|_{2}$, with increasing number of synergies.

A measure of the quality of the grasp (given in terms of the norm of contact forces needed to avoid slippage) is enhanced by increasing the number of actuated synergies, but only to a limited extent. No improvement is observed beyond the first three synergies in the precision grasp case, while continuous but small improvements are obtained in the whole-hand grasp case.

All the above results are consistently robust with respect to different values of stiffness parameters, which may reflect the uncertainty by which these parameters are known in human or robotic hand models, and/or the fact that grasp stiffness may be changed either voluntarily or not.

These results are consistent with the hypothesis that the first few postural synergies observed in grasp pre-shaping [1] are also crucial in grasping force optimization, when suitably translated from the kinematic configuraton space where they have been observed, to the force domain through the procedures illustrated in this paper.

\section{APPENDIX}

With reference to Fig. 1, and by employing the definition of the adjoint operator $\mathrm{Ad}_{(R, d)}$ given in [22], the contribution of the $i^{t h}$ finger, $j^{\text {th }}$ limb to the components in $\mathrm{P}$ of the wrench $w_{e} \in \mathbb{R}^{6}$ exerted on the object is given by

$$
{ }^{p} w_{e_{i j}}=G_{i j} f_{i j}, \quad G_{i j}:=\operatorname{Ad}_{\left({ }^{c_{i j}} R_{p}, d_{c_{i j}}{ }\right)} H_{i j}^{T} \in \mathbb{R}^{6 \times c_{i j}},
$$

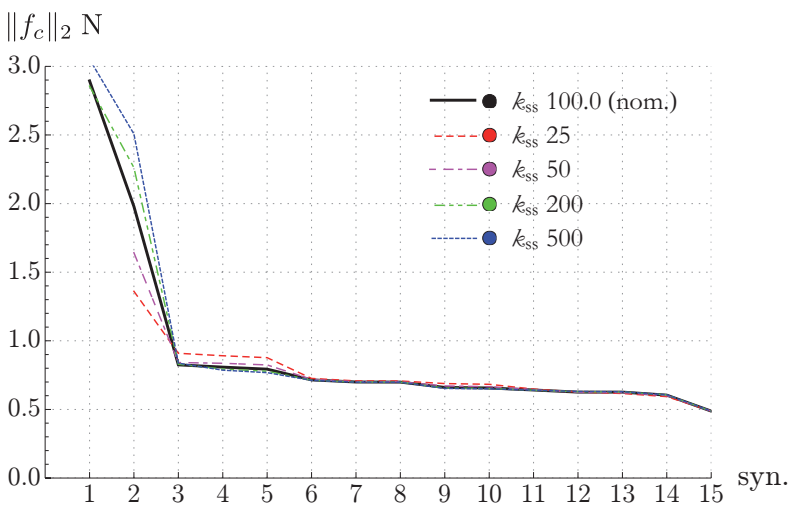

(a) Trends for variation of the steady-state gain $k_{\mathrm{ss}}(\mathrm{Nmm} / \mathrm{rad})$.

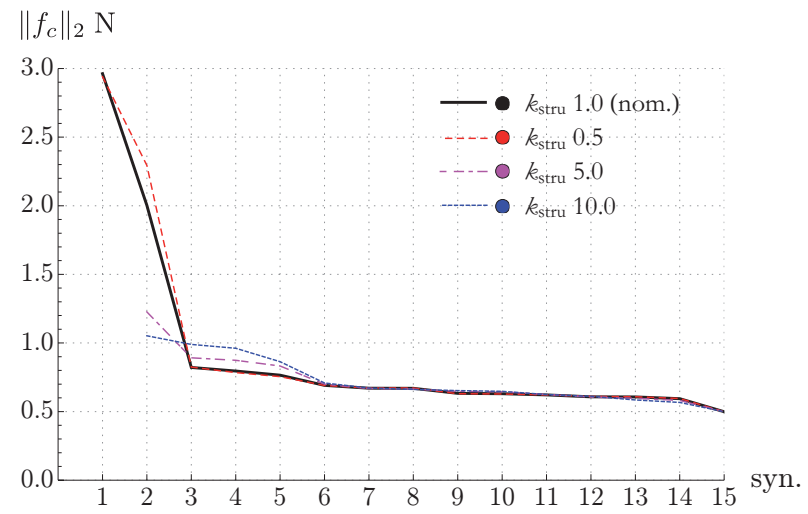

(b) Trends for variation of the structural stiffness $k_{\text {stru }}(\mathrm{N} / \mathrm{mm})$.

Fig. 7: The Ashtray. Norm of the optimal contact force $f$ w.r.t. the cost function $\|f\|_{2}$, with increasing number of synergies.

where ${ }^{c_{i j}} R_{p}$ is the rotation that aligns $\mathrm{P}$ onto $\mathrm{C}_{i j}$, and $d_{c_{i j}}^{e}$ is the vector from $C_{i j}$ to $E$. Let $\epsilon_{i j}$ be defined as follows

$$
\epsilon_{i j}= \begin{cases}1, & \text { if } j \in \nu(i) \\ 0, & \text { otherwise. }\end{cases}
$$

Put $f_{i}=\left[\epsilon_{i_{1}} f_{i_{1}}^{T} \cdots \epsilon_{i n_{i}} f_{i n_{i}}^{T}\right]^{T} \in \mathbb{R}^{c_{i}}$, where $c_{i}=$ $\sum_{\text {as }}{ }_{j \in \nu(i)} c_{i j}$, and consider the contribution of the $i^{t h}$ finger

$$
{ }^{p} w_{e_{i}}=G_{i} f_{i}, \quad G_{i}:=\left[\epsilon_{i 1} G_{i 1} \cdots \epsilon_{i n_{i}} G_{i n_{i}}\right] \in \mathbb{R}^{6 \times c_{i}}
$$

Stacking equation (37) for each finger, we can write the global grasp matrix as

$$
{ }^{p} w_{e}=G f, \quad G:=\left[G_{1} \cdots G_{k}\right] \in \mathbb{R}^{6 \times c},
$$

with $c=\sum_{i=1}^{k} c_{i}$.

Similarly, let $\xi_{c_{o_{i j}}} \in \mathbb{R}^{c_{i j}}$ be the local components in the constrained directions at point $c_{o_{i j}}$ due to a twist of the object ${ }^{p} \xi_{e}$, with components in P. By setting $\xi_{o_{i}}=$ $\left[\epsilon_{i 1} \xi_{c_{o_{i 1}}}^{T} \cdots \epsilon_{i n_{i}} \xi_{c_{o_{i n_{i}}}}^{T}\right]^{T} \in \mathbb{R}^{c_{i}}$, for the $i^{\text {th }}$ finger, and $\xi_{o}=$ $\left[\xi_{o_{1}}^{T} \cdots \xi_{o_{k}}^{T}\right]^{T} \in \mathbb{R}^{c}$, for the whole hand, we can write by duality

$$
\xi_{o}=G^{T p} \xi_{e}, \quad G^{T} \in \mathbb{R}^{c \times 6}
$$

We now consider the relationships for the statics and kinematics of the fingers. By employing the D.-H. convention, the 


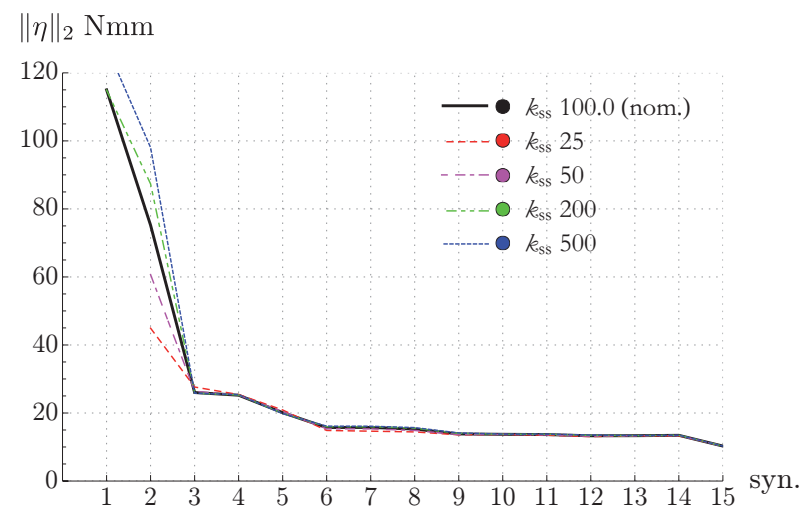

(a) Trends for variation of the steady-state gain $k_{\mathrm{ss}}(\mathrm{Nmm} / \mathrm{rad})$.

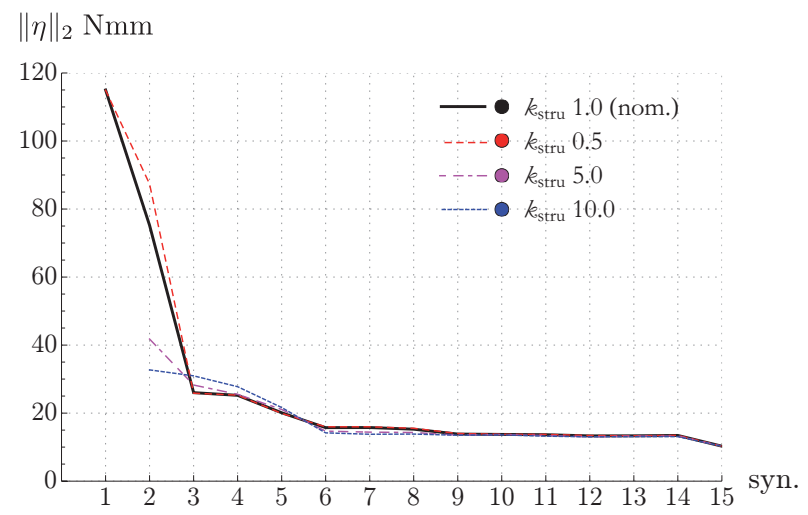

(b) Trends for variation of the structural stiffness $k_{\text {stru }}(\mathrm{N} / \mathrm{mm})$.

Fig. 8: The Ashtray. Norm of the optimal synergistic force $\eta=S^{T} \tau$ w.r.t. the cost function $\|\eta\|_{2}$, with increasing number of synergies.

local components in the constrained directions of the contact point $c_{f_{i j}}$ on the $i^{t h}$ finger, $j^{\text {th }}$ limb, can be written as

$$
\xi_{c_{f_{i j}}}=J_{i j} \dot{q}_{i}, \quad J_{i j}:=H_{i j} \operatorname{Ad}_{\left({ }^{c_{i j} R_{p}}, d_{c_{i j} o_{i j}}\right)} J_{o_{i j}}\left(q_{i}\right),
$$

where $J_{o_{i j}}=\left[\begin{array}{llllll}j_{i 1} & \cdots & j_{i j} & 0 & \cdots & 0\end{array}\right] \in \mathbb{R}^{6 \times n_{i}}$, with blocks defined as $(1 \leq l \leq j)$

$j_{i l}= \begin{cases}{\left[\begin{array}{ll}z_{l-1}^{T} & 0^{T}\end{array}\right]^{T},} & \text { for prismatic } l^{\text {th }} \text { joint } \\ {\left[\begin{array}{ll}\left(d_{o_{i j}}^{o_{i, l-1}}\right)^{T} \widehat{z}_{l-1} & z_{l-1}^{T}\end{array}\right]^{T},} & \text { for revolute } l^{t h} \text { joint }\end{cases}$

By stacking (40) for all the limbs on the $i^{\text {th }}$ finger that are actually in contact, and setting $\xi_{f_{i}}=$ $\left[\epsilon_{i 1} \xi_{c_{f_{i 1}}}^{T} \cdots \epsilon_{i n_{i}} \xi_{c_{f_{i n_{i}}}}^{T}\right]^{T} \in \mathbb{R}^{c_{i}}$, we obtain

$$
\xi_{f_{i}}=J_{i} \dot{q}_{i}, \quad J_{i}:=\left[\epsilon_{i 1} J_{i 1}^{T} \cdots \epsilon_{i n_{i}} J_{i n_{i}}^{T}\right]^{T} \in \mathbb{R}^{c_{i} \times n_{i}}
$$

Then, collecting the complete set velocities in twist $\xi_{f}=$ $\left[\xi_{f_{1}}^{T} \cdots \xi_{f_{k}}^{T}\right]^{T} \in \mathbb{R}^{c}$, yields

$$
\xi_{f}=J \dot{q}, \quad J:=\operatorname{Blockdiag}\left(J_{1}, \ldots, J_{k}\right) \in \mathbb{R}^{c \times n}
$$

Finally, again by duality arguments, the map from the hand contact forces $f$ to hand joint torques $\tau$ is given by

$$
\tau=J^{T} f, \quad J=\operatorname{Blockdiag}\left(J_{1}^{T}, \ldots, J_{k}^{T}\right) \in \mathbb{R}^{n \times c}
$$

\section{ACKNOWLEDGMENT}

Authors wish to gratefully acknowledge Marco Santello for the inspiring discussions and for providing experimental data and parameters. This work is supported by the European Commission under CP grant no. 248587, "THE Hand Embodied", within the FP7-ICT-2009-4-2-1 program "Cognitive Systems and Robotics".

\section{REFERENCES}

[1] M. Santello, M. Flanders, and J. F. Soechting, "Postural hand synergies for tool use," J. Neurosci., vol. 18, no. 23, pp. 10 105-10 115, December 1998.

[2] C. R. Mason, J. E. Gomez, and T. J. Ebner, "Hand synergies during reach-to-grasp," J Neurophysiol, vol. 86, no. 6, pp. 2896-2910, December 2001 .

[3] V. Cheung, A. d'Avella, M. Tresch, and E. Bizzi, "Central and sensory contributions to the activation and organization of muscle synergies during natural motor behaviors," Journal of Neuroscience, vol. 25, no. 27, pp. 6419-6434, 2005.

[4] A. Bicchi, "Hands for dextrous manipulation and robust grasping: a difficult road towards simplicity," IEEE Trans. on Robotics and Automation, vol. 16, no. 6, pp. 652-662, December 2000.

[5] M. Ciocarlie, C. Goldfeder, and P. Allen, "Dimensionality reduction for hand-independent dexterous robotic grasping," in Proceedings of the IEEE/RSJ International Conference on Intelligent Robots and Systems, 2007, pp. 3270-3275. [Online]. Available: http: //dx.doi.org/10.1109/IROS.2007.4399227

[6] C. Brown and H. Asada, "Inter-finger coordination and postural synergies in robot hands via mechanical implementation of principal component analysis," in IEEE-RAS International Conference on Intelligent Robots and Systems, 2007, pp. 2877-2882.

[7] M. T. Ciocarlie and P. K. Allen, "Hand posture subspaces for dexterous robotic grasping," The International Journal of Robotics Research, vol. 28, no. 7, pp. 851-867, July 2009.

[8] R. Featherstone, Rigid Body Dynamics Algorithms, Springer, Ed. Springer, 2008.

[9] D. Prattichizzo and J. C. Trinkle, "Grasping," in Springer Handbook of Robotics. Springer, 2008

[10] A. Bicchi, "On the problem of decomposing grasp and manipulation forces in multiple whole-limb manipulation," Robotics and Autonomous Systems, vol. 13, no. 2, pp. 127-147, July 1994.

[11] M. Cutkosky and I. Kao, "Computing and controlling the compliance of a robotic hand," Robotics and Automation, IEEE Transactions on, vol. 5, no. 2, pp. 151-165, 1989.

[12] A. G. Feldman and M. F. Levin, "The equilibrium-point hypothesispast, present and future," Adv. Exp. Med. Biol., no. 629, pp. 699-726, 2009.

[13] H. Hanafusa and H. Asada, "Stable prehension by a robot hand with elastic fingers," in $7^{\text {th }}$ ISIR, Tokyo, 1997.

[14] J. Joh and H. Lipkin, "Lagrangian wrench distribution for cooperating robotic mechanisms," in IEEE Conf. on Robotics and Automation, 1991

[15] I. Kao, M. Cutkosky, and R. Johansson, "Robotic stiffness contro and calibration as applied to human grasping tasks," Robotics and Automation, IEEE Transactions on, vol. 13, no. 4, pp. 557-566, 1997.

[16] J. Friedman and T. Flash, "Task-dependent selection of grasp kinematics and stiffness in human object manipulation," Cortex, no. 43, pp. 444460, 2007.

[17] A. Bicchi, "On the closure properties of robotic grasping," The International Journal of Robotics Research, vol. 14, no. 4, pp. 319-334, August 1995.

[18] M. Buss, H. Hashimoto, and J. B. Moore, "Dextrous hand grasping force optimization," IEEE Trans. on Robotics and Automation, vol. 12, no. 3, pp. 406-418, June 1996

[19] M. Grant and S. Boyd, "Cvx: Matlab software for disciplined convex programming (web page and software)," http://stanford.edu/ boyd/cvx, 2004.

[20] - "Graph implementations for nonsmooth convex programs," in Recent Advances in Learning and Control, 2008, pp. 95-110.

[21] K. C. Toh, M. Todd, and R. Tutuncu, "Sdpt3 - a matlab software package for semidefinite programming," Optimization Methods and Software, vol. 1, pp. 545-581, 1999.

[22] R. M. Murray, Z. Li, and S. Sastry, A Mathematical Introduction to Robotic Manipulation. Boca Raton, FL: CRC Press, 1994 